\title{
Raphael Thorius' Hymnus Tabaci: literarisches Spiel, medizinischer Traktat oder politisches Manifest?
}

\section{Tabakliteratur in der Frühen Neuzeit}

„Der Tabak hat eine weltgeschichtliche Bedeutung. Jeder muss ihm das zuerkennen, ganz einerlei, ob er ihn für gesund oder schädlich hält, ihn liebt oder verabscheut.“ Mit diesen Worten beginnt Hoffmann von Fallersleben seinen Traktat zum Tabak in der deutschen Litteratur. ${ }^{1}$ In der Tat wird man diese Äußerung als nicht übertrieben ansehen, wenn man bedenkt, dass der Tabak bald nach seinem Bekanntwerden in Europa nicht nur zu einem festen Bestandteil der Alltagskultur wurde, sondern auch eine beträchtliche wirtschaftliche Bedeutung erlangte. Auch die Naturwissenschaften beschäftigten sich mit ihm. Denn die neu importierte Pflanze musste in das sich etablierende System der Botanik integriert, ${ }^{2}$ ihre Qualitäten beschrieben und ihr medizinischer Nutzen erforscht werden. ${ }^{3}$ Zudem entzündeten sich am Tabak bald auch moralische, ja gar theologische Fragen, die ausgehandelt werden mussten. So stellte sich beispielsweise die im christlichen Kontext keineswegs belanglose Frage, ob der Tabakkonsum mit dem Fastengebot konfligierte. ${ }^{4}$

Angesichts der hohen Aktualität des Tabaks in der Frühen Neuzeit verwundert es nicht, dass dieses neu entdeckte Heil- und Genussmittel auch in der Literatur deutliche Spuren hinterlässt und in der Frühen Neuzeit Phasen zu konstatieren sind, in denen vielleicht nicht, wie Hoffmann von Fallersleben

1 Heinrich Hoffmann von Fallersleben: Der Tabak in der deutschen Literatur. In: Weimarisches Jahrbuch für die Deutsche Sprache, Litteratur und Kunst 2 (1855), S. 243-260, hier S. 243.

2 Vgl. David Harley: The Beginnings of the Tobacco Controversy: Puritanism, James I, and the Royal Physicians. In: Bulletin of the History of Medicine 67,1 (1993), S. 28-50, hier S. 29.

3 Das Verdienst der Erstbeschreibung kommt Nicolás Bautista Monardes mit dessen 1565 veröffentlichter Historia medicinal zu (lateinische Übersetzung des Carolus Clusius im Jahr 1574 als De simplicibus medicamentis ex occidentali India delatis).

4 Vgl. hier etwa den Traktat De sumptione del (sic!) Tabaco, quando exigitur naturae ieiunium ante sacram communionem des spanischen Moraltheologen Tomás Hurtado (1589-1659) in Thomás Hurtado: Tractatus varii resolutionum moralium [...]. Pars posterior. Lyon 1651, S. 188-223. 
schreibt, „jedes Blatt nach Tabak riecht“, ${ }^{5}$ der Tabak aber eine doch starke Präsenz aufweist.

Einen beeindruckenden Überblick über die Literaturproduktion zum Thema Tabak bietet der Katalog der Arents Tobacco Collection. Diese geht auf die Sammlung George Arents Jr. (1875-1960) zurück, der eine durchaus bemerkenswerte Persönlichkeit gewesen zu sein scheint: ${ }^{6}$ Spross einer durch Tabak reich gewordenen Familie, versuchte er sich nicht nur als Rennfahrer (und war dabei im Jahr 1904 in den ersten tödlichen Unfall der Vanderbild Cup Races verwickelt), sondern auch als Buchsammler:

In 1895, when a very young man, I decided to start a collection of books and was given good advice: not to buy some incunabula, seventeenth-century plays and nineteenthcentury novels and essays and think I was forming a collection of books. The result would be that I would have a lot of books, but not a real library. I should confine myself to buying books and manuscripts relating to one subject. As my family, for three generations, had been interested in the tobacco industry, it was a natural subject for me to choose. ${ }^{7}$

Arents erweiterte seine Büchersammlung zum Thema Tabak über die Jahre mit beachtlichem Engagement. Die Sammlung sucht, was Quantität und Qualität ihrer Objekte anbelangt, ihresgleichen. Die 4200 Objektbeschreibungen im von Jerome E. Brooks besorgten fünfbändigen Katalog der Arents Tobacco Collection erlauben einen, wenn vielleicht nicht vollständigen, so auf jeden Fall repräsentativen Überblick über die Tabakliteratur insgesamt. ${ }^{8}$

Das in Brooks' Katalog skizzierte mare magnum der Tabakliteratur ist bis heute kaum aufgearbeitet. ${ }^{9}$ Beschränkt man sich auf die lateinischen Texte, die keineswegs den geringsten Teil der (frühneuzeitlichen) Tabakliteratur ausmachen,

5 Hoffmann von Fallersleben (wie Anm. 1), S. 243. Für Hoffmann von Fallersleben, der sich mit dem Tabak in der deutschen Literatur beschäftigt, erstreckt sich dieser Zeitraum von ca. $1690-1730$.

6 Vgl. zu ihm H. George Fletcher: Indomitable Collector: Goerge Arents, Jr. and The New York Public Library. In: Biblion: The Bulletin of The New York Public Library 9 (2000), S. 87-103.

7 Vorwort zu Brooks, Nill (siehe folgende Anm.), Bd. 5.

8 Tobacco. Its History Illustrated by the Books and Manuscripts in the Library of George Arents, Jr. Hg. von Jerome E. Brooks (4 Bde.). New York 1937-1952, Bd. 5, hg. von Anne M. Nill, New York 1952, Nachdruck aller fünf Bände New York 1999.

9 Die vorliegenden umfassenderen Arbeiten zur Geschichte des Rauchens sind insgesamt eher anekdotischer Natur. Nützlich und bequem zugänglich sind z. B. Egon Caesar Conte Corti: Geschichte des Rauchens. Frankfurt a.M. 1930 (zahlreiche weitere Auflagen) oder aber die Monographie des schon genannten Jerome E. Brooks (The Mighty Leaf. Tobacco Through the Centuries. London, Sydney 1953). 
ist die Forschungslage sehr übersichtlich. ${ }^{10}$ Selbst die zwei wohl bedeutendsten Texte der neulateinischen Literatur zum Thema Tabak, der Hymnus Tabaci (begonnen um 1609, veröffentlicht 1625) des in London wirkenden flämischen Arztes Raphael Thorius und die Satyra contra abusum tabaci (1657) des Jesuiten Jacob Balde, sind nur sehr vereinzelt behandelt worden. ${ }^{11}$

Dieser Beitrag nimmt sich Thorius' Hymnus Tabaci an, der als literarisch ambitioniertes, gattungstypologisch komplexes und in seiner Intention polyvalentes Lehrgedicht ohne Frage größere Aufmerksamkeit verdient, als ihm bislang zuteil geworden ist.

\section{Raphael Thorius' Hymnus Tabaci}

Der im frühen siebzehnten Jahrhundert in England entstandene Hymnus Tabaci stellt einen ersten Höhepunkt der neulateinischen Tabakliteratur dar. Sein Autor, Raphael Thorius, ${ }^{12}$ in Flandern geboren - sein genaues Geburtsjahr ist unbekannt -, studierte in Oxford Medizin und arbeitete seit 1591 als Arzt in London. ${ }^{13}$ $\mathrm{Zu}$ seinen Patienten gehörte nach seiner Ankunft in England im Herbst 1610 bis zu seinem Tod im Jahr 1614 beispielsweise kein Geringerer als Isaac Casaubonus. Thorius erwarb sich somit auch indirekt in seiner Funktion als Arzt große

10 Einen bibliographischen Überblick über die neulateinische Tabakliteratur geben Dirk Sacré: Quid poetae scriptoresve de tabaco senserint vel scripserint. In: Vox Latina 22 (1986), S. 540-545 und ders.: De tabaco quid senserint vel scripserint scriptores neolatini: Auctarium. In: Vox Latina 25 (1989), S. 88-90.

$11 \mathrm{Zu}$ Thorius vgl. Ian D. McFarlane: Tobacco - A Subject for Poetry. In: From Wolfram and Petrarch to Goethe and Grass. Studies in Literature in Honour of Leonhard Forster. Hg. von D. H. Green, L. P. Johnson, Dieter Wuttke. Baden-Baden 1982, S. 427-441 und Christine Harrauer: Wer entdeckte tatsächlich den Tabak? Mythisches Erzählen bei Raphael Thorius und Jakob Balde. In: Antiker Mythos erzählt und angewandt bis in die Gegenwart. Symposion Wien 15.-17. November 2001. Hg. von Joachim Dalfen, Christine Harrauer. Wien 2004, S. 157-180. Thorius' Text ist digital ediert (vgl. Anm. 19), Baldes Tabaksatire liegt in einer modernen Ausgabe mit Übersetzung und Kommentar vor (Jacob Balde: Satyra contra abusum tabaci. Satire wider den Tabakmissbrauch. Hg. und übs. von Alexander Winkler. Mainz 2015).

$12 \mathrm{Zu}$ Thorius' Leben knapp Ole Peter Grell: Thorius, Raphael (d. 1625). In: Oxford Dictionary of National Biography 2004. https://doi.org/10.1093/ref:odnb/27336 (28. Mai 2020).

13 Seine Promotion erfolgte jedoch in Leiden. In der Leidener Universitätsbibliothek hat sich ein auf den 12.1.1591 datierter Druck seiner Theses medicae de Hydrope erhalten (Signatur: ASF 347: 60, non vidi). 
Verdienste um die bonae litterae. Der kränkelnde Casaubonus schätzte Thorius' medizinischen Rat sehr. ${ }^{14}$

Von Thorius' dichterischer Produktion erscheint zu seinen Lebzeiten lediglich seine In obitum Io. Barclaii elegia (London 1621) als eigenständige Publikation. ${ }^{15}$ Andere Werke sind nur handschriftlich überliefert. ${ }^{16}$ Der hier interessierende Hymnus Tabaci erscheint zusammen mit seinem eindrucksvollen Winter-Gedicht Hyems $^{17}$ nahezu zeitgleich mit Thorius' Tod bei Elsevier in Leiden mit einem verspielten, Bacchus und seinen qualmenden Tross der Satyrn und Mänaden darstellenden Frontispiz. ${ }^{18}$ Ein Jahr später veröffentlicht der Londoner Drucker John Haviland die beiden Werke auch in England. ${ }^{19}$

14 Vgl. etwa Casaubonus’ Tagebucheintrag zum „XVI. Kal. Mai“ des Jahres 1614: „Apud Meiernium virum clarissimum hodie pransus sum cum Thorio medico eruditissimo quorum consiliis, quae de mea valetudine inierunt, benedic, O Pater.“ (zit. nach Isaac Casaubonus: Ephemerides. Hg. von John Russel. Bd. 2. Oxford 1850, S. 1050-1051). Zwei Briefe Thorius’ an Casaubonus sind ediert in Paul Botley, Máté Vince (Hg.): The Correspondence of Isaac Casaubon in England. Bd. 1. Genf 2018 (1611 05 26, [1611 07] 00). Thorius hält die Umstände von Casaubonus’ Tod in der Epistola de viri celeberrimi Isaaci Casavboni morbi mortisqve cavsa (Leiden 1619) fest.

15 Ein an den Autor gerichtetes Liminalgedicht aus Thorius' Feder findet sich zudem in Matthias de L'Obel: In G. Rondelletii [...] Officinam Animadversiones. London 1605, fol. [థा 3r].

16 Die wichtigste bislang bekannt gewordene Handschrift stellt ohne Frage Sloane MS 1768 (non vidi) der British Library (London) dar, die zahlreiche dichterische Versuche enthält. Für eine Inhaltsübersicht der Handschrift vgl. Paul Bergmans: Les poésies manuscrites de François et Raphaël Thorius. In: Mélanges Paul Thomas: Recueil de mémoires concernant la philologie classique, dédié à Paul Thomas. Brügge 1930, S. 29-38.

17 Der Hyems erscheint erstmals als Annex zur 1625er Ausgabe des Hymnus Tabaci (zu den Ausgaben vgl. Anm. 19).

18 Thorius war mit dem Titelkupfer sehr zufrieden. So schreibt er am 26.2.1625 an van Kinschot: „Picturatam frontem [sc. das Titelkupfer] vidi, et risi inventoris ingenium“ (abgedruckt in der Ausgabe Leiden 1625, S. 46-47 des Hymnus Tabaci [vgl. Anm. 19]).

19 Raphael Thorius: Hymnus tabaci. Leiden 1625; Raphael Thorius: Hymnus tabaci. London 1626. Die gelegentlich zu findende Erwähnung einer Ausgabe Leiden 1628 geht auf eine falsche Interpretation der auf dem Frontispiz der 1625er Ausgabe missverständlich zu lesenden Jahresangabe zurück, vgl. Charles Pieters: Annales de l'imprimerie des Elsevier, ou Histoire de leur famille et de leurs éditions. 2. Aufl. Gent 1858, S. 27-28. Weitere Ausgaben des Hymnus: 1644 Utrecht (mit eigenem Titelblatt, doch unter fortgesetzter Paginierung Giles Everards De herba panacea [...] und anderen tabakistischen Werken beigebunden, der Hymnus Tabaci auf S. 225296), 1651 London (Editio Nova, Multò Emendatior), London 1716 (unter dem Titel Tabacum. Poema libris duobus. Hg. von Henry Player, der auch eine englische Überstzung des Hymnus Tabaci veröffentlichte) und 1800 Lissabon (De Paeto seu Tabaco carminum libri duo. Hg. von José Mariano de Conceição Vellozo). Der Hymnus findet sich auch in verschiedenen Auflagen der Musae Anglicanae (Oxford 1692, S. 245-292; Oxford 1699, Bd. 1, S. 245-292; London 1714, Bd. 1, S. 245-292; London 1721, Bd. 1, S. 220-263; London 1741, Bd. 1, S. 190-225; London 1761, Bd. 1, S. 190-225). 2012 hat Mark Riley eine „hypertext edition“ des Hymnus Tabaci vorgelegt 
Der ersten Ausgabe sind zwei Briefe vorangestellt. Zunächst der chronologisch spätere, vom Herausgeber des Hymnus Tabaci, Lodewijk van Kinschot (1595-1647), ${ }^{20}$ an den Leser adressierte (fol. A2r-A3r), in dem van Kinschot angibt, dass er zwei Jahre zuvor ein unvollständiges und fehlerhaftes Manuskript des Hymnus (venustissimum de Paeto opus [...] distortum, neque parva sui parte mutilum) erhalten habe. Er habe den Autor um eine bessere Abschrift gebeten und $\mathrm{zu}$ seiner Freude von Thorius eine überarbeitete und erheblich erweiterte Version des Hymnus (exemplar, partim ad incudem revocatum, partim media plus parte auctum) zugeschickt bekommen. Der zweite Brief ist Thorius' Begleitschreiben zur Übersendung des besagten Manuskripts (fol. A3v-[A4r]). In diesem beschreibt der Autor die Erweiterung des Hymnus etwas genauer: Mitto igitur priorem Hymnum correctum: cui, adaucta supellectile, secundum adiunxi. Während die Details der Werkgenese im Dunkeln liegen, kann doch immerhin festgehalten werden, dass der Hymnus Tabaci ursprünglich auf ein Buch angelegt war und von Thorius später auf zwei Bücher erweitert wurde. Da Thorius im eben genannten Brief vom 18.2.1625 angibt, der Hymnus sei bereits 16 Jahre alt, so muss das erste Buch auf das Jahr 1609 datiert werden. Ob die Erweiterung des zweiten Buchs, das immerhin fast 800 Verse umfasst, tatsächlich erst auf van Kinschots Anfrage hin um 1624/1625 zustande kam oder aber von Thorius bereits zuvor ausgearbeitet worden war, ist fraglich.

Der Hymnus Tabaci ist, wie aus dem Proöm hervorgeht, Sir William Paddy gewidmet:

Tu qui censu decoratus Equestri

Virtutem titulis, titulos virtutibus ornas,

Antiquum et Phoebi nato promittis honorem,

Tu, Paddaee, fave: nec enim praestantior alter

Morbifugae varias vires agnoscere plantae,

Inque tubo genitas haurire et reddere nubes. ${ }^{21}$

$(1,3-8$, S. 1)

(http://www.philological.bham.ac.uk/thorius/; am 7.12.2019 überprüft, mit der WaybackMachine des Internet Archive archiviert und somit auch über den Permalink https://web.archive. org/web/20191207131454/http://www.philological.bham.ac.uk/thorius/ einsehbar).

$20 \mathrm{Zu}$ ihm W. M. C. Regt: Kinschot (Mr. Louis of Lodewijk van). In: Nieuw Nederlandsch Biografisch Woordenboek. Leiden 1937. Bd. 10, Sp. 463-464.

21 Der Text des Hymnus Tabaci folgt dem der Erstausgabe Leiden 1625 (wie Anm. 19). Die Versangabe erfolgt nach der Online-Edition Rileys (vgl. Anm. 19), die Seitenangaben beziehen sich auf die Erstausgabe Leiden 1625. Ligaturen wurden in der Transkription aufgelöst, , v' wurde, wenn es sich um einen Vokal handelt, mit , $u^{‘}$ transkribiert, $j^{j}$ regelmäßig mit ,í. Die Interpunktion sowie Groß- und Kleinschreibung folgen dem Druck. Alle Übersetzungen, sofern nicht anders angegeben, stammen vom Autor. 
$\mathrm{Du}$, der du in den Ritterstand erhoben wurdest und deine Tugend mit Titeln, die Titel aber mit deinen Tugenden zierst, und Aeskulap, dem Sohn des Phoebus Apollo, seine ursprüngliche Ehre zurückzugeben versprichst, du, Sir Paddy, sei uns gewogen! Niemand nämlich erkennt besser die verschiedenen Kräfte dieser Heilpflanze und niemand kann die in der Pfeife entstehenden Wolken besser schlürfen und wieder ausatmen.

William Paddy (1554-1634), ${ }^{22} 1603$ von König James geadelt - hierauf ist mit censu decoratus Equestri angespielt - war einer der angesehensten Mediziner seiner Zeit und unter anderem von 1609 bis 1611, im wahrscheinlichen Entstehungszeitraum des Kerns des Hymnus Tabaci, Präsident des Londoner College of Physicians, welchem auch Thorius angehörte. Wenn Thorius Sir Paddy als hervorragenden Tabakkenner und versierten Raucher apostrophiert, so ist dies nicht bloße Rhetorik. Paddy war 1605 anlässlich eines Besuchs des Königs in Oxford mit seiner medizinischen Expertise in Sachen Tabak in Erscheinung getreten. Er argumentierte - obgleich selbst Raucher - bei einer Debatte über die Frage An creber suffitus Nicotianae exoticae sit sanis salubris ${ }^{23}$ vor dem König in einer offenbar recht launigen Rede öffentlich gegen den Tabak. ${ }^{24} 1620$ wurde er zudem vom König zum Mitglied einer Kommission ernannt, die über die Qualität des in England verkauften Tabaks wachen und die Eintreibung der Steuern kontrollieren sollte. Zum Zeitpunkt der Überarbeitung des Hymnus Tabaci war Thorius' Kollege und alter Bekannter also einer der höchsten Wächter über den englischen Tabakhandel und somit geradezu zum Widmungsadressaten prädestiniert.

22 Zu ihm vgl. Lauren Kassell: Paddy, Sir William (1554-1634). In: Oxford Dictionary of National Biography 2004. https://doi.org/10.1093/ref:odnb/21080 (28. Mai 2020) und Donald S. Pady: Sir William Paddy, M.D. (1554-1634). In: Medical History 18,1 (1974), S. 68-82.

23 Nach Harley (wie Anm. 2), S. 46 Anm. 52. Ein Echo dieser Debatte könnte die in den mythologischen Kontext des Trojanischen Kriegs übertragene, von Thorius wohl fingierte Debatte „plantae de laudibus Indae“ (1,340, S. 13) zwischen dem Brüderpaar Podalyrius und Machaon darstellen. Beide vertraten „amicis [...] odiis“ (1,339-340, S. 13) entgegengesetzte Meinungen, obwohl sie beide den Tabak schätzten und vor der Debatte selbst auch geraucht hatten. Dass Paddy während seiner Rede vor dem König rauchte, schreibt Anthony Nixon: Oxford Triumph [...]. London 1605, fol. C2v.

24 Vgl. ebd.: „In one of these questions [sc. „Whether the often taking of Tobacco bee wholesome for such as are sound and in health?"] Doctor Paddy being answerer, held against Tobacco, being a great drinker of Tobacco himselfe.“ Die positive Reaktion des Königs auf Paddys Rede wird ebd. beschrieben. 


\section{Literarische Struktur}

Zunächst soll der Inhalt des zwei Bücher sehr ungleicher Länge (366 und 746 Verse) umfassenden Hymnus Tabaci summarisch vorgestellt werden: ${ }^{25}$ Der namentlich nicht genannte, doch der Einfachheit halber mit Thorius zu identifizierende Sprecher erzählt von Bacchus' sagenhafter Indienkampagne, ${ }^{26}$ auf der nur die zufällige Entdeckung ${ }^{27}$ des Tabaks Bacchus' Tross vor dem sicheren Tod durch Hunger und Durst rettet und ihm kurz darauf sogar zum Sieg über die ,indischen' Ureinwohner verhilft. Nach dieser ätiologischen Erzählung von der Entdeckung des Tabaks bespricht der Erzähler im Rest des ersten Buchs die botanischen Qualitäten und die medizinischen Wirkungen des Tabaks. Im zweiten Buch wird die mythologische Erzählung wiederaufgegriffen: Bacchus, jetzt Herr über Indien, will die dort lebenden wilden Völker zivilisieren. Er sucht einen der berüchtigtsten Häuptlinge ([r]egulus, 2,54, S. 17) namens Haematoës

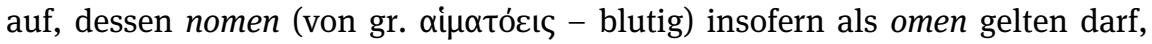
als er vom Erzähler in grotesker Überzeichnung als Erzkannibale dargestellt wird. Mit Wein kann Bacchus sich bei Haematoës Gehör verschaffen. Bacchus, der über einen medizinisch geschulten Blick verfügt, diagnostiziert ihm einen desolaten Gesundheitszustand und empfiehlt den Tabak als Heilmittel. Hierauf setzt Silen, Bacchus' rechte Hand, zu einer langen Ausführung über den medizinischen Nutzen des Tabaks an, an deren Ende er - eine komische Volte - einschläft. Da seine Zuhörer jedoch noch wissen möchten, wie Tabak angebaut werden kann, übernimmt der Erzähler ersten Grades - Thorius - metaleptisch dort, wo Silen aufgehört hatte, und bringt das Gedicht mit Erläuterungen zum Tabakanbau zu Ende.

Die hexametrische Form, die didaktische Programmatik sowie nicht zuletzt auch der konkrete botanische, medizinische und agronomische Lehrgehalt lassen den Hymnus Tabaci spontan als Lehrgedicht erscheinen. Die knappe

25 Einen ausführlicheren Überblick über den Inhalt bietet Mark Riley: Introduction. In: Raphael Thorius, Hymnus Tabaci (1626). A hypertext edition. 2012. http://www.philological.bham.ac.uk/ thorius/intro.html, §§ 9-14 (28. Mai 2020).

26 Bacchus' Indienzug, wie ihn prominent der spätantike griechische Epiker Nonnos in seinen Dionysiaka (48 Gesänge, fünftes Jahrhundert) erzählt, kann in Amerika spielen, weil der Hymnus Tabaci nicht zwischen India orientalis und India occidentalis unterscheidet.

$27 \mathrm{Zu}$ der hier gebotenen Ätiologie im Vergleich zu anderen Berichten vgl. Harrauer (wie Anm. 11). Jacob Balde wird in der Fabula de herba tabaci (Sylvae VIII, 6, in: Jacob Balde: Opera omnia. München 1729. Bd. 2, S. 240-244) seine eigene Ätiologie ebenfalls mit Bacchus’ Eroberungsfeldzug durch Indien in Verbindung bringen. 
Inhaltsangabe $\mathrm{zu}$ Beginn $^{28}$ sowie der mit [a]ggredior ${ }^{29}$ markierte Auftakt im Proöm der Dichtung greifen zudem unmissverständlich Muster der antiken Lehrdichtung auf:

Innocuos calices, et amicam vatibus herbam,

Vimque datam folio, et laeti miracula fumi

Aggredior.

$$
(1,1-3, \text { S. 1) }
$$

Ich mache mich daran, die unbescholtenen Becher, das den Dichtern liebe Kraut, die dem Blatt gegebene Kraft und die Wunder des beglückenden Rauchs zu besingen.

Nach dem Proöm setzt jedoch die Handlung ganz im Zeichen des Epos ein. Wenn auch das Epyllion im neulateinischen Lehrgedicht durchaus seinen festen Platz hat, ${ }^{30}$ ist im Hymnus Tabaci die mythologische Erzählung von Bacchus’ Indienkampagne für das Werk geradezu konstitutiv. Sie stellt das eigentliche narrative Gerüst dar, in das die didaktischen Passagen eingebunden werden. Der Sprecher tritt nicht wie im Lehrgedicht üblich primär als Lehrer auf, sondern als epischer Erzähler. Wiederholt finden sich intradiegetische lehrgedichtartige Passagen, die von einer homodiegetischen Erzählinstanz präsentiert werden. So wird der mit 450 Versen längste zusammenhängende Sinnabschnitt des Hymnus Tabaci, in dem vom richtigen Gebrauch, vom Nutzen und von den Gefahren des Tabaks gehandelt wird, Silen in den Mund gelegt. Auf den Sprecher selbst entfallen lediglich zwei didaktische Passagen: die botanische, pharmakologische und medizinische Diskussion des Tabaks (1,222-359, S. 9-14) und die Ausführungen zum Anbau der Tabakpflanze (2,644-720, S. 39-41) - insgesamt gut 200 Verse.

Auf das Epos verweisen auch einige intertextuelle Merkmale: In der Erzählung vom Eroberungszug der Bacchanten wendet sich der Anführer - Bacchus mit $O$ socii $(1,45, \mathrm{~S}$. 2$)$ an seine Gefährten und verwendet dabei genau die Anrede, mit der auch Aeneas im ersten Buch der Aeneis $(1,198)$ seine Leute anspricht.

28 Prominentestes klassisches Beispiel für eine Inhaltszusammenfassung am Beginn eines Lehrgedichts sind Vergils Georgica.

29 Vgl. Verg. georg. 1,5: „hinc canere incipiam“, oder auch Lucr. 1,55: „disserere incipiam“. Den Gestus hatte auch schon Fracastoro in seiner Syphilis aufgegriffen („hinc canere [...] incipiam“, Syph. 1,10-12). „Aggredior“ verwendet Lukrez lexikalisch äquivalent zu „incipiam“ z. B. in 6,940 .

30 Vgl. hierzu zumindest Walther Ludwig: Neulateinische Lehrgedichte und Vergils Georgica. In: From Wolfram and Petrarch to Goethe and Grass. Studies in Literature in Honour of Leonhard Forster. Hg. von D. H. Green, L. P. Johnson, Dieter Wuttke. Baden-Baden 1982, S. 151-180 und Heinz Hofmann: Aristaeus und seine Nachfolger: Bemerkungen zur Rezeption des Aristaeus-Epyllions in der neulateinischen Lehrdichtung. In: Humanistica Lovaniensia 52 (2003), S. 343-398. 
Bacchus wir hierdurch mit dem Protagonisten der Aeneis parallelisiert - eine Verbindung, die durchaus plausibel wirkt, da ja auch im Hymnus Tabaci Bacchus als fürsorglicher Anführer und Eroberer fungiert. Überdies weist der vom Erzähler ersten Grades (d. h. ,Thorius') knapp paraphrasierte Lehrvortrag, mit dem Silen seine Mitbacchantinnen und -bacchanten nach durchstandener Not unterhält (1,134141, S. 6), deutliche Parallelen zum Gesang des Iopas in der Aeneis (1,742-746) auf. In beiden Fällen waren die Akteure einer existenziellen Bedrohung entronnen: die Aeneaden dem Seesturm, die Anhänger des Bacchus dank des Tabaks dem Tod durch Hunger und Durst. In beiden Fällen findet die gelöste Stimmung ihren Ausdruck in einem Mahl, das mit einem Lehrvortrag ausklingt. Der sermo, den Thorius seinen Silen halten lässt, ist dem Lied des Vergilischen Iopas dabei obgleich parodistisch verzerrt - recht ähnlich. Wenn Iopas in einem weihevollen Auftritt mit wallendem Haar und goldener Lyra bekanntermaßen von Sonnenfinsternissen, Sternbildern, Wetterphänomenen und dem Ursprung von Mensch und Tier singt, liegt Silen paffend im Schatten einer Tabakstaude und breitet, ganz offensichtlich durch den Tabak intellektuell besonders angeregt, zum Zwecke der gepflegten Unterhaltung sein umfassendes Wissen aus:

Tum vacuus densa Paeti porrectus in umbra,

Omnigenûm ut patuit faecunda scientia rerum,

Dulcibus absumit lentas sermonibus horas,

Explicat ingentes opulentae mentis acervos,

Ludenti et similis Naturae arcana recludit,

Implicitos memorat sphaeris caelestibus orbes,

Compagemque hominis, terrae et miracula pandit,

Addit et oraclis docti libamina fumi.

$(1,134-141$, S. 6)

Dann legte er sich sorglos in den dichten Schatten des Tabaks. Als sich ihm das beredte Wissen um allerhand Dinge eröffnet, bringt er lange Stunden mit angenehmen Reden zu. Er entfaltet den mächtigen Inhalt seines reichen Gedächtnisses, wie im Spiel eröffnet er die Geheimnisse der Natur. Er erwähnt die in die Himmelssphäre eingefassten Planetenbahnen und erklärt den Bau des Menschen und die Wunder der Erde. $\mathrm{Zu}$ diesen bemerkenswerten Reden fügt er Weihegaben des gelehrten Rauchs.

Dass hier der Spaß und die Geselligkeit im Vordergrund stehen, zeigt nicht zuletzt auch die explizite Angabe, Silen trage seine Weisheit „fast wie im Spiel“ ([l]udenti [...] similis) vor. Der Erzähler unterstreicht die heitere Lockerheit durch ein bewusst überzogenes Register, wenn er von den Ausführungen als oracl[a] spricht, die noch dazu nahezu im Wortsinn „,beweihräuchert“ werden.

Ein auf ähnliche Weise ironisch gebrochener Bezug auf den epischen Gattungsrahmen findet sich in der darauffolgenden Szene: Nachdem man die Rettung vor Hunger und Durst eine Nacht lang ausgiebig gefeiert hatte, rufen Bacchus und 
Silen den dionysischen Heereszug zum Kampf auf. Insbesondere sollten sie, so Bacchus, genügend Tabak mitführen. Dieser mache ihnen einerseits Mut, ${ }^{31}$ andererseits könnten die Feinde durch den Rauch eingeschüchtert werden. ${ }^{32}$ Der Aufmarsch von Bacchus' Trupp wird in pompöser epischer Form geschildert, während der naturgemäß wein- und tabaklastige Inhalt einen letztlich komischen Kontrast hierzu darstellt:

Vino acuunt iras $^{33}$ resides, haustoque Tabaco

Excludunt lethi faciem, ${ }^{34}$ suaque agmina circum

Nube tegunt atra: spirantes naribus ignem ${ }^{35}$

Procedunt: medio glomeratur in aequore nimbus

Igne micans, tonitruque fero, fumoque stupendus.

$(1,194-198$, S. 8)

Mit Wein schüren sie ihre träge Wut, mit dem Tabak halten sie sich die Fratze des Todes vom Leib und sie bedecken ihren Trupp ringsherum mit einer dunklen Wolke. Sie schreiten voran, indem sie aus der Nase Feuer atmen. Mitten auf dem Feld türmt sich eine Wolke auf, die von Feuer blitzt, staunenswert durch ihren wilden Donner und ihren Rauch.

Die Strategie geht auf: Die Indi packt schon beim Anblick des qualmenden Trupps die Angst und sie flüchten, ohne dass es zum Kampf kommt. Die mythologische Handlung wird nun zugunsten der durch den Erzähler ersten Grades präsentierten Ausführungen zur Zusammensetzung und Wirkung des Tabaks ausgesetzt und erst zu Beginn des zweiten Buchs wieder aufgegriffen. Gerade das erste Buch des Hymnus Tabaci weist, wie gezeigt wurde, markante epische Referenzen auf, die freilich mitunter parodistisch gebrochen werden. $\mathrm{Zu}$ Beginn des zweiten Buchs wird die mythische Handlung fortgesetzt. Der territorialen Eroberung Indiens folgt die kulturelle: Bacchus will das als barbarisch beschriebene ,Indien“ zivilisieren. Als Beispiel für dieses Bemühen ist ein Treffen mit einem indigenen Häuptling namens Haematoës geschildert. Diese zentrale Episode des zweiten Buchs ist ein in der Art eines Epyllions kohärenter, wenngleich nicht zu Ende erzählter Handlungsstrang. ${ }^{36}$ Für unseren Zusammenhang bedeutsam scheint hierbei abermals der Präsentationsmodus des eigentlichen Lehrgehalts des zweiten Buchs: Schon im ersten Buch hatte Silen als Erzähler zweiten Grades wiederholt kurze didaktische

31 Vgl. für diesen militärischen Effekt des Tabaks auch Baldes Tabaksatire (wie Anm. 11), § 26.

32 Die Rede des Bacchus in 1,160-181, S. 7.

33 Vgl. Aen. 9,464: „variisque acuunt rumoribus iras“.

34 Der Ausdruck „leti facies“ bei Luc. 3,653.

35 Vgl. Aen. 7,280: „spirantis naribus ignem“.

36 Nachdem der intradiegetische Erzähler (Silen) einschläft, führt der Erzähler ersten Grades seinen Lehrvortrag zwar zu Ende, kommt aber nicht mehr auf Haemotoës zurück. Es bleibt somit unklar, welcher Erfolg Bacchus' Zivilisierungsbemühungen beschieden ist. 
Beiträge geliefert, ${ }^{37}$ im zweiten Buch fällt ihm nun eine veritable Vorlesung über den Tabak zu. Es wird also auch hier ein seinem Umfang und Gehalt nach zentraler Lehrinhalt auf eine untergeordnete Erzählebene verschoben. ${ }^{38}$ Der primäre Erzähler ähnelt im Hymnus somit vielmehr dem Erzähler im Epos als dem gerne in erster Person Wissen vermittelden Lehrer des Lehrgedichts. ${ }^{39}$ Es zeigt sich hier also die gattungstypologische und strukturelle Fluidität des Lehrgedichts, ${ }^{40}$ bei dem man es nach Wilhelm Kühlmann oftmals mit „offenen, nicht selten strukturell synkretistischen Textformaten zu tun hab[e]““41

\section{Die Poetisierung der Wissenschaft}

Van Kinschot erwähnt in seinem Einleitungsbrief mit Fracastoros Syphilis einen für den Hymnus Tabaci relevanten Referenztext. Dies gilt weniger im Sinne

37 Die Mahnrede gegen den ungezügelten Tabakkonsum in 1,76-104 (S. 4-5) sowie der oben bereits besprochene ,universale‘ Lehrvortrag (1,134-141, S. 6).

38 Nur am Rande sei ein kleiner Fehler in dieser Verschachtelung der Erzählebenen notiert. In 2,354-355 verweist der Erzähler zweiten Grades (Silen) auf eine zuvor von ihm gemachte Feststellung („Hunc ergo iubeo in mentem revocare, quod olim / Sanximus [...]“). Dieser intratextuelle Verweis bezieht sich auf die Darstellung der mitunter konträren Wirkungen des Tabaks in 1,223-225 (S. 9-10), die dort jedoch vom Erzähler ersten Grades geboten wird. Diese Inkonsequenz könnte schlicht auf einen lapsus memoriae des Autors zurückgehen, der das zweite Buch in einem großen zeitlichen Abstand zum ersten schreibt. Ebenso wäre denkbar, dass Teile der Rede des Silen in einer früheren Überarbeitungsstufe des Gedichts vom Erzähler ersten Grades gesprochen wurden. Thorius hätte es dann nach der Modifikation versäumt, die nötigen Anpassungen vorzunehmen.

39 In der Terminologie der Scaligerianischen Poetik verwendet Thorius also das epische „mixtum“ und nicht die der Lehrdichtung angemessene „narratio simplex“, vgl. Wilhelm Kühlmann: Wissen als Poesie. Ein Grundriss zu Formen und Funktionen des frühneuzeitlichen Lehrgedichts im deutschen Kulturraum des 16. und 17. Jahrhundert. Berlin, Boston 2016, S. 10 mit dem Verweis auf die Poetik Scaligers (Hg. von Luc Deitz, Gregor Vogt-Spira. Stuttgart-Bad Cannstatt 1994. Bd. 1, S. 90-91).

40 Ein Indiz für die Schwierigkeit der gattungsmäßigen Einordnung des Hymnus mag der Umstand sein, dass er in der (wohlgemerkt kargen) Forschung mit unterschiedlichen Etiketten versehen wurde. Sarah Dickson: Panacea Or Precious Bane: Tobacco in Sixteenth-century Literature. Dissertation New York University 1954, S. 203 nennt den Hymnus Tabaci - freilich ohne weitere Erläuterung - ein „epyllion on tobacco“, McFarlane (wie Anm. 11), S. 430 bringt den Hymnus in einer bewusst vorsichtigen Formulierung mit Vergils Georgica in Verbindung („There is a vague hint of Thorius writing a sort of Georgics on the cultivation of tobacco“). Riley (wie Anm. 25), § 9 spitzt McFarlanes Aussage auf problematische Weise zu, indem er den Hymnus Tabaci schlechterdings und weniger treffend als ,the Georgics of tobacco cultivation“ bezeichnet.

41 Kühlmann (wie Anm. 39), S. 2. 
einer ausgeprägten lexikalischen oder strukturellen Intertextualität, sondern vielmehr auf der abstrakteren Ebene der inventio: Wie Fracastoro mit dem morbus Gallicus, so wählte auch Thorius ein recht unattraktives Thema für sein Lehrgedicht. Van Kinschot sieht in der gekonnten Bearbeitung eines schwierigen, ja womöglich für Dichtung auf den ersten Blick ungeeigneten Stoffs eine besondere Leistung. Thorius habe es wie Fracastoro geschafft, sich „aus einem nahezu unrühmlichen Stoff Ruhm zu erwerben“ (ex argumento prope infami famam sibi peperit, fol. A2v). Diese literarische ,Veredelung“ oder ,Poetisierung des Gegenstands geschieht dabei auf inhaltlich-struktureller und auch sprachlicher Ebene: Inhaltlich-strukturell gilt es den Stoff in ein ansprechendes Narrativ einzubinden. Dies erreicht Thorius insbesondere durch den wiederholten Rekurs auf die Mythologie und die Verwendung verschiedener Erzählebenen. Sprachlich stellt die keineswegs triviale Verbindung technisch korrekter und präziser Fachterminologie mit den Anforderungen metrisch gebundener und stilistisch befriedigender Diktion eine bedeutende Herausforderung dar, wie auch aus den im Folgenden zu besprechenden Beispielen hervorgeht.

Die Rede von der ,Poetisierung“ der Wissenschaft lässt sich jedoch noch aus einer weiteren Perspektive betrachten. Sie wird bei Thorius geradezu zur erkenntnisermöglichenden Methode stilisiert. Beim lehrdichterischen Bravourstück des Hymnus, der pharmakologischen Analyse der Wirkprinzipien des Tabaks (1,222290, S. 9-12), beschreibt Thorius zunächst die enorme Schwierigkeit, die Ursachen für die advers[ae] [...] virtut[es] (1,223, S. 9), d. h. die teils sehr verschiedenen, mitunter auch konträren Wirkungen des Tabaks, ${ }^{42} \mathrm{zu}$ ergründen. Da nicht zu entscheiden sei, ob der Tabak seiner Substanz nach als simplex oder compositum angesehen werden müsse (1,235-242, S. 9-10), wählt Thorius, um einen Ausweg aus dieser Aporie zu finden, ein diskursives Verfahren, bei dem er teils deduktiv teils induktiv vorgeht: Durch die Betrachtung von exempla, unter denen einerseits naturwissenschaftliches Wissen, andererseits aber auch empirische Beobachtungen verstanden werden, entwickelt er einen Gedankengang, mit dem er sich der Wahrheit, so gut es geht, anzunähern hofft:

Si tamen exemplis libeat, velut indice filo,

Ire per anfractus dubios errore viarum,

Inque sibi similes Totum dissolvere partes,

Forsitan occurret, quo mens placata quiescat,

Et si non verum, at veri illucescat imago.

$(1,243-247$, S. 10)

42 So könne der Tabak z. B. sowohl stopfend als auch abführend wirken, vgl. 1,342-365, S. $27-28$. 
Wenn es aber beliebt, anhand von konkreten Beispielen, gleichsam wie an einem den Weg weisenden Faden entlang, durch schwer zu überblickende verwinkelte Straßen zu irren und das Ganze in sich ähnelnde Teile aufzulösen, dann wird uns vielleicht etwas einfallen, was den Geist befriedet und ruhen lässt. Es wird dann wenn nicht die Wahrheit selbst, so doch ein Abbild der Wahrheit erstrahlen.

Beim Tabak ist der Geruch besonders markant. Für Geruch und Geschmack sind Salze verantwortlich. Daher beginnt Thorius eine schulbuchartige Taxonomie der Salze, die sich ihrer Natur nach in salia fixa und salia volatilia unterteilen lassen (1,250-256, S. 10). Bei dieser Ausführung kommt ihm - so die Fiktion einer mimetischen, im Moment statthabenden Erforschung des vorgestellten Sachverhalts in den Sinn (Hic aliud memori occurrit ratione tenendum, 1,257, S. 10), dass jede Pflanze die jeweils ihrer Natur zukommenden Nährstoffe aus der Erde bezieht und damit auch deren Qualitäten übernimmt. Thorius präsentiert nun sein Wissen über diverse im Boden enthaltene Substanzen, von denen er in einer virtuos formulierten Passage sieben (Schwefel, Nitrum, Arsenik, Bitumen/Naphtha, Quecksilber, Vitriol, Alaun) auf effektiv vier Verse verdichtet:

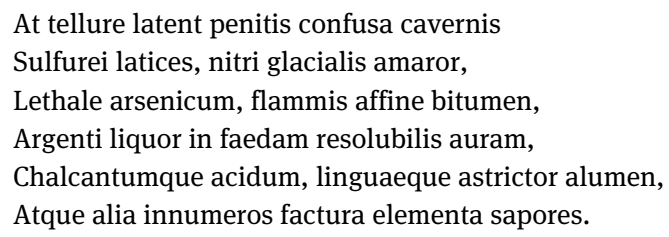

Doch unter der Erde sind tief in Höhlen verborge Schwefelströme, das kalte und bittere Nitrum, das tödliche Arsenik, das dem Feuer verwandte Bitumen, das in gräßliche Luft sich auflösende Quecksilber, das saure Vitriol und der Alaun, der einem die Zunge zusammenzieht, und andere Elemente, die unzählige Geschmäcker hervorbringen.

Aus dieser Zusammenstellung scheint er besonders die Qualitäten des bitumen im Tabak wiederzuerkennen, der u. a. „fett, stark riechend, beißend und zäh“ sei und „das Feuer liebe“ (vgl. 1,273-274, S. 11). Auch der empirisch-haptische Befund und die Wirkung des Tabaks auf den Körper deute auf bitumen hin: dextram / Trita oleo faedat, digitos et glutine viscat (1,277-278, S. 11).

Thorius inszeniert auf diese Weise im Medium der Dichtung einen wissenschaftlichen, d.h. rational aus gesichertem Wissen und empirischer Erfahrung ableitenden Erkenntnisprozess und ermöglicht dem Leser/der Leserin dessen simultanen Nachvollzug. Die enge Verquickung von poetischer Präsentation und wissenschaftlichem Erkenntnisstreben findet ihren prägnanten Ausdruck in der Phrase, mit der Thorius die eben skizzierte Suche nach dem wesentlichen Bestandteil des Tabaks beginnt: Si quis iam excutiat prolixo carmine, de tot / Principiis, cui accepta ferat sua munera Paetum [...] (1,269-270, S. 11). 
Hier wird das (Lehr-)Gedicht (carmen) auch in sprachlicher Hinsicht geradezu zu einem Instrument der Erkenntnissuche gemacht. Der Hymnus Tabaci weist durch die Inszenierung des wissenschaftlichen Erkenntnisprozesses Züge einer nicht bloß belehrenden, sondern gar ,forschenden“ Dichtung auf, was den wissenschaftlichen Anspruch des Textes unterstreicht. Denn ungeachtet seiner verspielten, bald parodierenden, bald ironisierenden Form und der in den Paratexten bemühten Bescheidenheitstopik, die den Hymnus als Spielerei ${ }^{43}$ und den Tabak als nicht vollends ernstzunehmenden Gegenstand für ein Gedicht (argumentum quidem leve ac ludicrum, so van Kinschot, fol. A2v) darstellt, ist Thorius' Text durchaus auch ein respektabler Beitrag zum botanisch-medizinischen Tabakdiskurs, der sich mit den für das frühe siebzehnte Jahrhundert einschlägigen, mit dem Tabak befassten Traktaten messen kann. Nicht ohne Grund, so scheint es, findet Thorius' Gedicht in dem 1644 in Utrecht als bibliographische Einheit konzipierten und publizierten Sammelband zum Tabak neben Giles Everards De herba panacea (erstmals 1587) und Johann Neanders Tabacologia (erstmals 1622) seinen Platz. ${ }^{44}$

\section{Der Hymnus Tabaci als politisches Manifest?}

Das Thema Tabak war unter der Regentschaft James' I. ein brisantes Politikum der König höchstpersönlich hatte ihn 1604 in seinem A Counterblaste to Tobacco verteufelt - und Gegenstand hitziger Debatten. Tabakrauchen war zu Beginn des siebzehnten Jahrhunderts insbesondere in London zur teils begeistert aufgenommenen, teils heftig kritisierten Mode geworden. ${ }^{45}$ Das Meinungsspektrum zum Tabak reicht dabei von kategorischer Ablehnung über die Verteidigung gemäßigten Tabakkonsums zu therapeutischen Zwecken bis hin zur liberalen Verteidigung des Rauchens. ${ }^{46}$

Wie sich Thorius in dieser Debatte positioniert, geben schon die ersten Verse des Proöms zu verstehen (vgl. Zitat oben S. 102). Es wird darin eine Abhandlung in

43 Thorius schreibt in seinem Brief an van Kinschot (fol. A3v in der Ausgabe Leiden 1625 [wie Anm. 19]) von seinen „lusus“ und nennt sich einen „ludentem inter pueros senem“.

44 Dass der Sammelband (wie Anm. 19), der zudem noch die lateinische Übersetzung von James' I. Counterblaste enthält (Misocapnus, sive de abusu tabaci lusus regius), trotz des den einzelnen bibliographischen Einheiten vorangestellten Titelblatts eindeutig als Einheit konzipiert ist, zeigt die durchgehende Paginierung.

45 Für eine knappe Skizze der Londoner Tabakszene (und deren Kritiker) vgl. Brooks (wie Anm. 9), S. 66-74.

46 Vgl. hierzu Brooks (wie Anm. 9), S. 67-70. 
Aussicht gestellt, die die positiven Eigenschaften und die wundersamen Segnungen des Tabaks behandelt. Der Hymmus verschweigt zwar keineswegs auch mögliche Risiken des Tabaks, ${ }^{47}$ doch könnten diese durch den richtigen Gebrauch (vgl. iustos sumendi [...] usus in 2,313, S. 26) gebannt werden. Gewöhnlich gegen den Tabak ins Feld geführte Vorwürfe ${ }^{48}$ werden entkräftet, manche Kritikpunkte werden jedoch auch geteilt. So wendet sich Thorius - über sein intradiegetisches Sprachrohr Silen - gegen all diejenigen, die nur aus modischen Gründen rauchten, sich um Nutzen und Schaden des Tabaks jedoch wenig kümmerten. ${ }^{49}$

Sunt qui fumum ideo, ut potent tantummodo, potant, Urbanos inter ne non habeantur amoeni, Prositne an noceat sibi susque deque ferentes[.]

$(2,303-305$, S. 26)

Manche rauchen nur des Rauchens wegen, damit diese feinen Gestalten auch ja zu den Modischen gehören. Sie kümmern sich dabei kein bisschen darum, ob das Rauchen nützt oder schadet.

Thorius verfolgt mit seinem Hymnus ein auf konstruktiven Disput ausgelegtes und somit im besten Sinne akademisches Programm. Er habe, schreibt er am Ende des Gedichts, seine Meinung dargelegt und lade alle, die nicht mit ihm übereinstimmen, zur begründeten Widerrede ein:

Si quis amans veri nobis dissenserit, author Consilii alterius, quod pectore claudit, in albo Scribat, et hos sensus recta ratione refellat.

$(2,744-746$, S. 42)

Wenn jemand aus Wahrheitsliebe mit uns nicht übereinstimmt, dann möge der Vertreter einer anderen Meinung publik machen, was er auf dem Herzen hat, und diese meine Meinung mit vernünftigen Argumenten widerlegen.

Diese Position ist gewiss intellektuell redlich, doch auch als Vorsichtsmaßnahme zu verstehen, steht Thorius mit seinen Ausführungen doch in einem deutlichen Gegensatz zu James’ I. Haltung. Vereinzelt scheint der Hymnus Tabaci dem Counterblaste rundheraus zu widersprechen.

47 Vgl. etwa die Silen in den Mund gelegten Ausführungen, für wen Tabakkonsum geeignet ist und für wen nicht, in 2,293-295, S. 26-27.

48 So schwäche der Tabak die Geistesschärfe (2,423 ff., S. 30-32) und mache impotent $(2,462-$ 464, S. 32-33).

$49 \mathrm{Zu}$ der Mode des ,recreational smoking، vgl. Brooks (wie Anm. 9), S. 66-70 und Harley (wie Anm. 2), S. 29-30. 
In diesem Zusammenhang interessiert insbesondere die Ursprungsgeschichte des Tabaks, die James und Thorius beide markant an den Beginn ihrer Texte stellen. In James’ Augen sei der Tabak von den „barbarous Indians“ als Heilmittel gegen die Syphilis (,a filthy disease“) ${ }^{50}$ entdeckt und vom ihm verhassten und bewusst nicht namentlich genannten Sir Walter Raleigh „by an inconsiderate and childish affectation of Noveltie“"51 nach England eingeführt worden. Für James bedeutet Tabakkonsum die Nachahmung barbarischer Gebräuche und somit die Infragestellung der eigenen zivilisatorischen Superiorität:

And now good Countrey men let us (I pray you) consider, what honour or policie can moove us to imitate the barbarous and beastly maners of the wilde, godlesse, and slavish Indians, especially in so vile and stinking a custome $?^{52}$

Durch die geschickte fiktionale Kontextualisierung der Entdeckung des Tabaks vermag ihn Thorius in ein Narrativ der westlichen Kulturgeschichte einzubinden. Von einer „corrupted basenesse of the first use of this Tobacco“ ${ }^{453}$ kann nach Thorius' Darstellung nicht die Rede sein. Dass die im Hymnus Tabaci gebotene Ätiologie gänzlich erfunden ist und dies von Thorius' Zeitgenossen auch so verstanden wurde, steht außer Frage. Viel relevanter ist jedoch die in der Erzählung vollzogene Fokusverschiebung: Der Tabak ist nicht nur „a common herbe, which [...] growes almost every where“,${ }^{54}$ der von vermeintlich Wilden zur Behandlung einer schmutzigen Krankheit verwendet wurde, sondern eine mächtige Heilpflanze, deren positives Potenzial sich erst durch kundige Anwendung entfalten kann, mithin Kultur voraussetzt und befördert.

Der politische Gehalt des Hymnus Tabaci beschränkt sich jedoch nicht auf die Auseinandersetzung mit James' Haltung zum Tabak. Vielmehr lässt sich die breiten Raum einnehmende Eroberungserzählung vor dem Hintergrund der englischen Kolonialpolitik lesen: $:{ }^{55}$ Im ersten Jahrzehnt des siebzehnten Jahrhunderts beginnt England auf dem amerikanischen Kontinent Fuß zu fassen. 1607, kurz bevor Thorius mit der Konzeption des Hymnus beginnt, gründet die Virginia Company of London mit Jamestown die erste koloniale Siedlung auf dem amerikanischen Kontinent. Die Parallele zur mythologischen Erzählung von der kolonisierenden Landnahme dürfte also durchaus erkennbar gewesen sein.

50 [James I]: A Counterblaste to Tobacco, London 1604, fol. B1v.

51 Ebd.

52 Ebd.

53 Ebd., fol. B2r.

54 Ebd., fol. B1v.

55 Harrauer (wie Anm. 11), S. 163 liest die mythologische Erzählung dagegen eher als „spielerische Spiegelung der ,Westindien'-Expedition des Columbus“. 
Bacchus, der Anführer des Eroberungszugs - und damit gewissermaßen alter ego James' I. - wird als milder Herrscher dargestellt, dem es nach erfolgreichem Abschluss seiner Kampagne in erster Linie um die Verbreitung von Zivilisation zu tun ist:

Ut sedit solio Victor, non tristibus armis

Saeviit in vitam vel opes, nec durus abactis

Indigenis statuit dominos, nec more tyranno

Optavit metui, metuit non ipse timentes;

Lenibus alloquiis, et maiestate remissâ

Partus amor populi, facilis clementia mores

Erudiit, glebamque iugo parere suasit.

$(2,21-27$, S. $15-16)$

Als er siegreich auf seinem Thron Platz genommen hatte, da wütete er nicht mit strenger Gewalt gegen das Leben und den Besitz, noch vertrieb er hartherzig die einheimischen Herren, um eigene einzusetzen. Er wollte auch nicht wie ein Tyrann gefürchtet werden und hatte daher auch selbst keine Furcht vor verängstigten Untertanen. Durch sanfte Ansprache und mit der zurückhaltenden Würde seines Rangs gewann er die Liebe des Volkes. Seine gutmeinende Milde verfeinerte deren Sitten und brachte den Boden dazu, sich dem Pflug zu fügen.

Das Verhältnis zwischen Eroberer und Eroberten ist ausgesprochen human. Thorius beschreibt es mit der paradoxen Wortverbindung „Freiheit in Abhängigkeit“ ([l]iberta[s] [...] serva, 1,209, S. 9) ${ }^{56}$. Aufgrund seiner Milde und Menschlichkeit genießt der Eroberer bei den Ureinwohnern höchstes Ansehen. Der Hymnus Tabaci darf vor diesem Hintergrund geradezu als zwischen Panegyrik und Handlungsempfehlung oszillierender (Kolonial-)Fürstenspiegel gelten, der den richtigen Umgang mit der Urbevölkerung aufzeigt. Bacchus lehrt die Ureinwohner insbesondere den Ackerbau (gleba[m] iugo parere suasit) und nach Silens ausführlicher Darlegung der Vorzüge des Tabaks sind die Indi wenig überraschend insbesondere am Anbau des Tabaks interessiert (2,635-638, S. 38). Durch dieses Wissen werden die einstmals unzivilisierten Völker zu Bauern (die im folgenden Zitat bezeichnenderweise ,coloni‘ genannt werden), die durch ihre Produkte, wie Thorius gegen Ende des Hymnus bemerkt, letztlich dem Handel und in Form von Steuern auch den Herrschern nützen:

merx ampla coloni

Augebit census, et vectigalia regum, Mercantum et loculos[.]

$(2,721-723$, S. 41)

56 Laut TLL 7,2,1318,17-18 ist diese Verbindung zuerst bei Petrus Chrysologus (fünftes Jahrhundert) belegt. 
Die üppige Ware wird vergrößern den Besitz des Bauern, die Steuereinnahmen der Könige und Einnahmen der Händler.

Das fiskalische Potenzial des Tabakhandels war James selbstverständlich nicht neu. Denn ungeachtet seiner feindlichen Haltung dem Tabak gegenüber versuchte der König seit Mitte der 1610er Jahre den Tabakhandel mit den amerikanischen Kolonien durch finanzpolitische Maßnahmen profitabler zu gestalten. ${ }^{57}$

Der Hymnus Tabaci gewinnt durch diese Bezugnahme auf den zeithistorischen Hintergrund auch eine politische Dimension. Er liefert aus medizinischer Perspektive eine energische Verteidigung des Tabaks und befreit diesen zudem vom Odium des Barbarischen, wodurch er James' grundsätzlicher Ablehnung des Tabaks entgegenwirkt. Die nahegelegte Parallelisierung von mythischer Erzählung und zeitgenössischem Geschehen bietet zudem ein gewisses panegyrisches Potenzial, wenngleich natürlich James I. gerade in seiner Darstellung als Entdecker und Befürworter des Tabaks allenfalls ein ironisches, wenn nicht vergiftetes Lob gesehen hätte.

Die im Titel des Beitrags gestellte Frage, ob es sich beim Hymnus Tabaci um ein literarisches Spiel, einen medizinischer Traktat oder ein politisches Manifest handelt, ist wohl am befriedigendsten so zu beantworten: Der Hymnus ist gleichermaßen ein durch Komik und Ironie einerseits und andererseits durch eine ansprechende sprachlich-strukturelle Darbietung unterhaltender, durch seine medizinischen und agronomischen Ausführungen belehrender und dabei auch die zeitgenössische Politik tangierender Text, der gerade durch diese Vielfältigkeit das große Ausdruckspotenzial neulateinischer Lehrdichtung zu illustrieren vermag.

57 Vgl. hierzu Donald A. Walker: Virginian Tobacco During the Reigns of the Early Stuarts: A Case Study of Mercantilist Theories, Policies, and Results. In: Mercantilist Economics. Hg. von Lars Magnusson. Boston u. a. 1993, S. 143-171. 